Optimized electrode coverage of membrane actuators based on epitaxial PZT thin films

This article has been downloaded from IOPscience. Please scroll down to see the full text article.

2013 Smart Mater. Struct. 22085013

(http://iopscience.iop.org/0964-1726/22/8/085013)

View the table of contents for this issue, or go to the journal homepage for more

Download details:

IP Address: 130.89.205.35

The article was downloaded on 11/07/2013 at 10:27

Please note that terms and conditions apply. 


\title{
Optimized electrode coverage of membrane actuators based on epitaxial PZT thin films
}

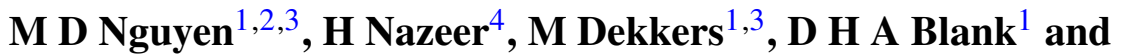 \\ G Rijnders ${ }^{1}$ \\ ${ }^{1}$ Inorganic Materials Science (IMS), MESA+ Institute for Nanotechnology, University of Twente, \\ PO Box 217, 7500AE Enschede, The Netherlands \\ ${ }^{2}$ International Training Institute for Materials Science (ITIMS), Hanoi University of Science and \\ Technology, No. 1 Dai Co Viet road, Hanoi, Vietnam \\ ${ }^{3}$ SolMateS B V, Drienerlolaan 5, Building 6, 7522NB Enschede, The Netherlands \\ 4 Transducers Science and Technology (TST), MESA+ Institute for Nanotechnology, \\ University of Twente, PO Box 217, 7500AE Enschede, The Netherlands
}

E-mail: d.m.nguyen@utwente.nl

Received 27 February 2013, in final form 10 June 2013

Published 11 July 2013

Online at stacks.iop.org/SMS/22/085013

\begin{abstract}
This research presents an optimization of piezoelectric membrane actuators by maximizing the actuator displacement. Membrane actuators based on epitaxial $\mathrm{Pb}(\mathrm{Zr}, \mathrm{Ti}) \mathrm{O}_{3}$ thin films grown on all-oxide electrodes and buffer layers using silicon technology were fabricated. Electrode coverage was found to be an important factor in the actuation displacement of the piezoelectric membranes. The optimum electrode coverage for maximum displacement was theoretically determined to be $39 \%$, which is in good agreement with the experimental results. Dependences of membrane displacement and optimum electrode coverage on membrane diameter and PZT-film/Si-device-layer thickness ratio have also been investigated.
\end{abstract}

(Some figures may appear in colour only in the online journal)

\section{Introduction}

In recent times, piezoelectric micro-pumps have been developed in a variety of applications, ranging from controlling and delivering tiny amounts of fluids on a lab-on-a-chip system to use in laboratory experiments [1], especially in drug delivery and biomedical applications [2,3]. Clearly, micro-pumps are one of the important microfluidic components where small size and accurate flow volume control are needed and, therefore, the design of micropumps plays an important role for practical applications of devices. To develop a suitable design of micro-pump for real-time applications it is very important to understand that these systems consist of many components, such as actuators, valves, chambers, nozzle diffuser mechanisms and appropriate pumping parameters [4-7]. In a 'normal' diaphragm pump, a piston moves a flexible diaphragm up and down for charging and discharging a liquid medium, displacing the medium pulse-wise. In a piezoelectric micro- pump, a piezoelectric element or piezoelectric actuator acts as the piston [8]. Therefore, the performance of micro-pumps depends strongly on the features of the piezoelectric membrane actuators [9-11].

Among piezoelectric materials, $\mathrm{Pb}(\mathrm{Zr}, \mathrm{Ti}) \mathrm{O}_{3}(\mathrm{PZT})$ is an attractive option for microelectromechanic systems (MEMS) technology due to its superior ferroelectric and piezoelectric properties [12]. In PZT materials, since bulk ceramic and thick film PZT are not suitable for miniature devices with low operating voltage, thin film technology has been widely used to prepare the submicron-thick PZT layer. Epitaxial growth of PZT thin films on a silicon substrate is considered to be a key technology for the fabrication of thinner and smaller electronic devices, because the leakage current is expected to be lower than that of polycrystalline films [13, 14]. Furthermore, epitaxial PZT films also exhibit much better ferroelectric and piezoelectric properties than polycrystalline PZT thin films [15]. 
The displacement of membrane actuators, based on PZT thin films and Si device layers, are controlled by many parameters: the growth technique, the thicknesses of the PZT thin-film and Si device layer, the membrane diameter and the top-electrode size. The membrane diameter can change the piezoelectric response, giving a high displacement and low resonance frequency for a large diameter. The thicknesses of the PZT film and Si device layer also have an influence on the piezoelectric properties, making it necessary to investigate these in order to improve the membrane performance.

The effect of the top-electrode size (or electrode coverage 'EC': the area ratio of the top-electrode to silicon membrane) on the piezoelectric responses has been described. For instance, Cho et al showed that an EC of $42 \%$ was predicted theoretically, while an EC of $60 \%$ was obtained from experiment, to give the maximum coupling coefficient $[16,17]$. However, in a recent paper [18] Choi et al showed that the coupling coefficient was maximized when the electrode coverage was about $25 \%$. This means that more studies are still necessary to understand the effect of electrode coverage on the piezoelectric responses of membrane actuators.

Concerning the dependence of the piezoelectric displacement of membranes on the electrode coverage there are a limited number of publications [19]. Therefore, in this paper, we have focused on the design, fabrication and characterization of membrane actuators based on epitaxial PZT thin films. The design was aimed at optimizing the piezoelectric response of the actuator membrane from the point of view of the top-electrode coverage. Pulsed laser deposition (PLD) of piezoelectric stack actuators based on thin films of $\mathrm{Pb}\left(\mathrm{Zr}_{0.52} \mathrm{Ti}_{0.48}\right) \mathrm{O}_{3}$ (PZT) sandwiched between oxide $\mathrm{SrRuO}_{3}$ (SRO) electrodes deposited on yttria-stabilized zirconia (YSZ) buffered Si substrates are presented in section 2.1. The ferroelectric properties are shown in section 2.2. The measurement technique used to define the piezoelectric responses is described in section 2.3. Finally, the relations between electrode coverage and the displacement and resonance frequency of the membrane actuator are discussed in section 3 .

\section{Experimental procedure}

\subsection{Design and fabrication of the piezoelectric micro-membranes}

2.1.1. Thin film fabrication. Compared with other common film deposition techniques, such as sputter deposition [20,21], evaporation [22, 23], metalorganic chemical vapor deposition (MOCVD) [24, 25] and sol-gel processing [26, 27], PLD has many important advantages for the epitaxial growth of oxide thin films. Most important is its ability to transfer material stoichiometrically from a multicomponent target to a growing film. This is very important for complex oxide-films such as PZT, because their physical properties depend strongly on the precise control of the chemical composition. The high supersaturation during each deposition pulse allows the growth of volatile thin film materials at high temperature. In the case of PZT thin film deposition, it results in a less significant loss of the more volatile elements, such as lead $(\mathrm{Pb})$.

Figure 1 shows the fabrication process of the piezoelectric membrane. Devices were fabricated on p-type (100) silicon-on-insulator (SOI) substrates (size: $20 \times 20 \mathrm{~mm}^{2}$ ) with a $5 \pm 0.2 \mu \mathrm{m}$-thick Si device layer, a $2 \pm 0.1 \mu \mathrm{m}$-thick buried-oxide (BOX) layer and a $380 \pm 20 \mu$ m-thick Si handle layer, as shown in figure 1(a). The deposition of a piezoelectric stack SRO/PZT/SRO/YSZ began with the deposition of a $100 \mathrm{~nm}$-thick YSZ buffer-layer on top of the SOI substrate (figure 1(b)). The YSZ buffer-layer prevents the formation of an excessive $\mathrm{SiO}_{2}$ amorphous layer on the surface of the Si-substrate, creating a crystallization template for the epitaxial growth of the piezoelectric stack. Next, a $100 \mathrm{~nm}$-thick SRO bottom electrode and a PZT layer $(0.5-3.0 \mu \mathrm{m})$ are deposited. Deposition of the piezoelectric stack is completed with a $100 \mathrm{~nm}$-thick SRO top-electrode layer. SRO is an ideal electrode in devices incorporating oriented ferroelectric films, due to its relatively high thermal conductivity and good compatibility in structure and chemistry with perovskite-type ferroelectric materials such as $\mathrm{PZT}, \mathrm{PbTiO}_{3}$ and $\mathrm{BaTiO}_{3}$ [28]. Moreover, conductive SRO oxide electrodes are known to reduce the fatigue characteristics in both the ferroelectric and piezoelectric displacement properties, through compensation of the oxygen vacancies near the electrode/film interface by changing their oxygen non-stoichiometry [29, 30].

From x-ray diffraction (XRD), the (110)-orientation is measured for PZT thin films grown on SRO/YSZ/Si substrates, as previously observed [30]. The epitaxial orientation relationship with the Si-substrate was obtained for all YSZ, SRO and PZT layers, described by PZT(110) ॥ SRO(110) ॥ YSZ(001) \| Si(001) [31].

\subsubsection{PiezoMEMS devices fabrication. Figure 1(c) and (d)} show the fabrication process of the piezoelectric membranes. First, the SRO top-electrode was etched by Ar-ion beam etching (figure 1(c)). Afterwards, the PZT film was sequential wet-etched and cleaned in diluted $\mathrm{HF}: \mathrm{HNO}_{3}: \mathrm{H}_{2} \mathrm{O}$ and $\mathrm{HCl}: \mathrm{H}_{2} \mathrm{O}$ solutions at specific areas to access the bottom electrode (see figure 1(c)). A lateral underetch of about 1-2 $\mu \mathrm{m}$ for a $1 \mu \mathrm{m}$ thick PZT layer was achieved. Then, the bottom SRO electrode and YSZ buffer-layer were also IBM etched. The etching rates are $10.0 \mathrm{~nm} \mathrm{~min} \mathrm{~min}^{-1}$ and $3.5 \mathrm{~nm} \mathrm{~min}-1$, respectively, for SRO and YSZ layers. Finally, the backside silicon hole was etched by deep reactive ion etching (DRIE) with the etching gases $\mathrm{SF}_{6}$ (etching cycle) and $\mathrm{C}_{4} \mathrm{H}_{8}$ (passivation cycle) to release the membrane actuator. The BOX layer that acts as an etch stop in the DRIE process was then removed using buffered-hydrofluoric acid (HF 12.5\%). A typical SEM image of the backside of a fabricated circular membrane actuator is shown in figure 2(a), in which the diameter corresponds to that of its design. The schematic cross-section and optical image (top-view) of a membrane actuator are shown in figures 1(e) and 2(d), respectively. 
(a)

(b)

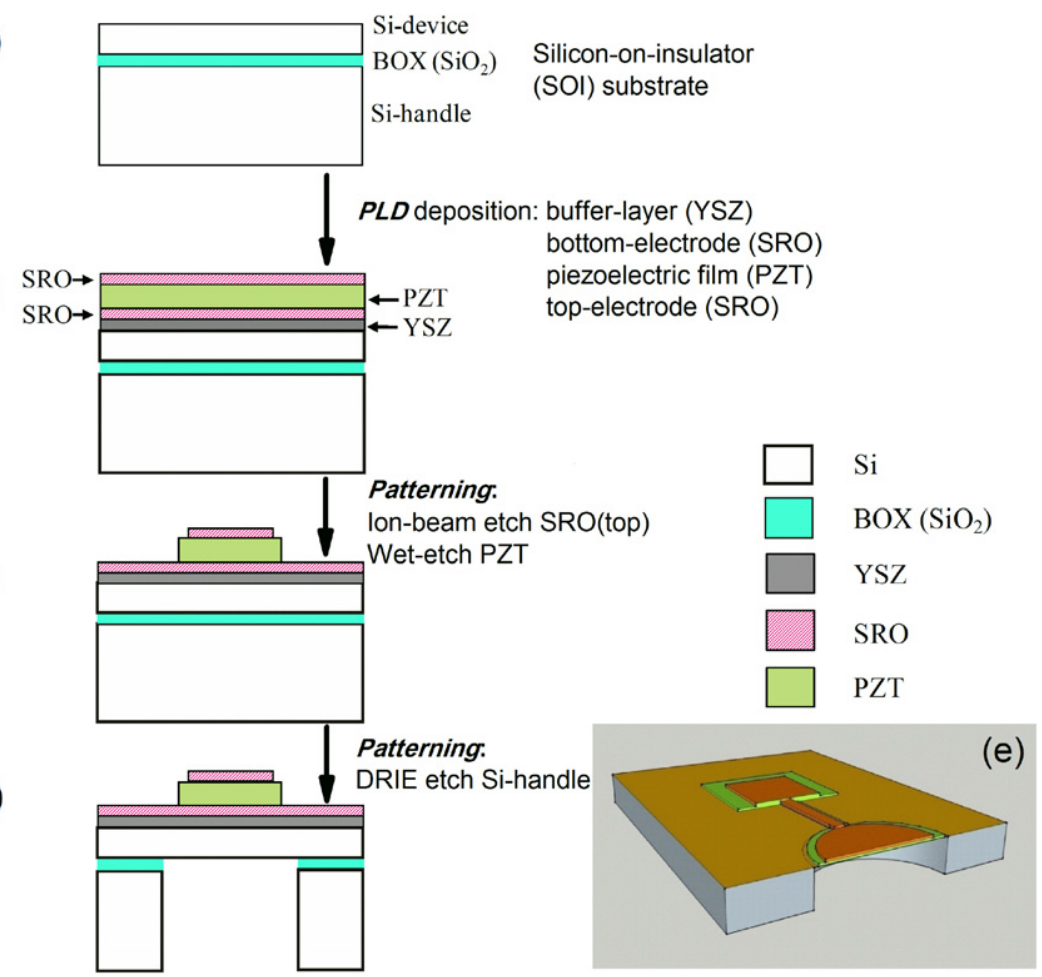

Figure 1. Schematic drawing of the fabrication process of a PZT thin film membrane actuator: (a) silicon-on-insulator (SOI) substrate, (b) PLD deposition of the piezoelectric stack (SRO/PZT/SRO/YSZ on SOI), (c) Ar-ion beam etching of the SRO top-electrode and the subsequent wet-etching of the PZT film, and (d) deep reactive ion etching (DRIE) of the Si handle layer. (e) Schematic cross-sectional view of a membrane actuator
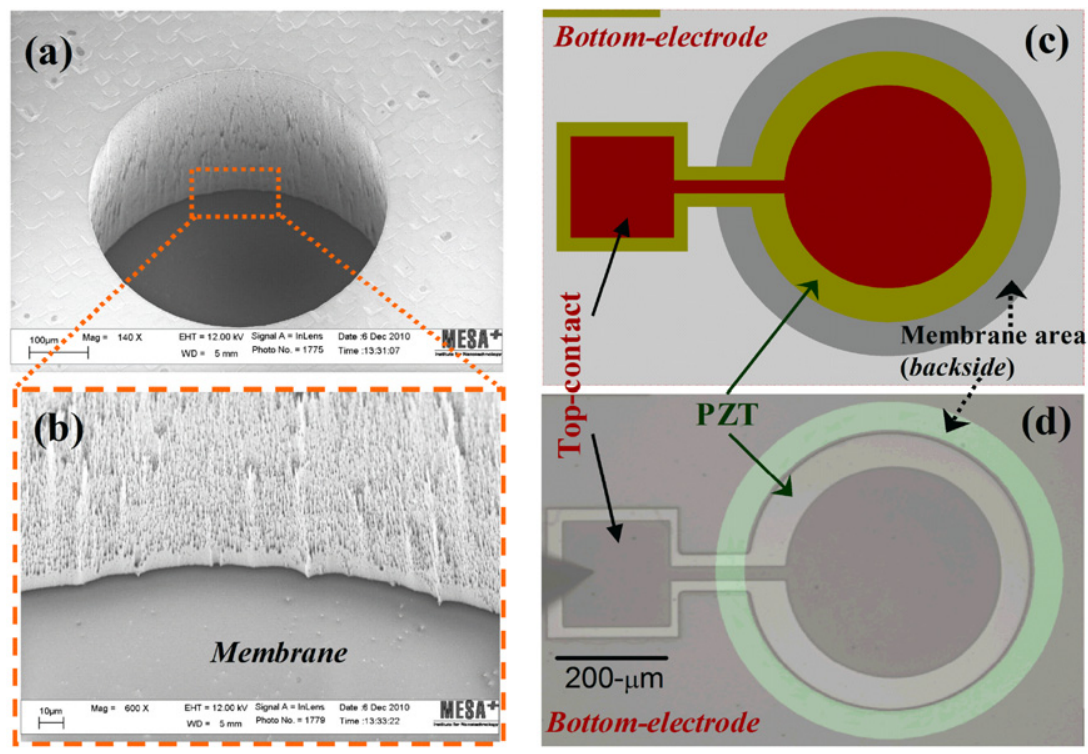

Figure 2. (a) SEM images of the backside view of the fabricated membrane actuator with a diameter of 500- $\mu \mathrm{m}$ and (b) zoomed-in image near the edge of the membrane, from the backside; (c) schematic detail of a designed membrane and (d) optical image (top-view) of a fabricated membrane actuator.

\subsection{Measurement of ferroelectric properties}

One of the key measurements for ferroelectric materials is, naturally, the measurement of the polarization hysteresis loop. The Sawyer-Tower circuit [32] is well-known as the (first) traditional hysteresis loop measurement setup (figure 3(a)), and this circuit has been modified to avoid noise by applying a virtual ground [33] (figure 3(b)). Nowadays, most testing of the ferroelectric capacitors is carried out using commercial apparatus available from one of two companies, Radiant Technologies and aixACCT. In this paper, the polarization hysteresis $(P-E)$ loop was measured at $\pm 200 \mathrm{kV} \mathrm{cm}^{-1}$ 


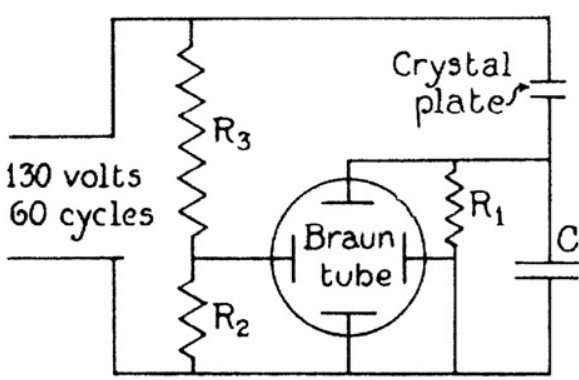

(a)

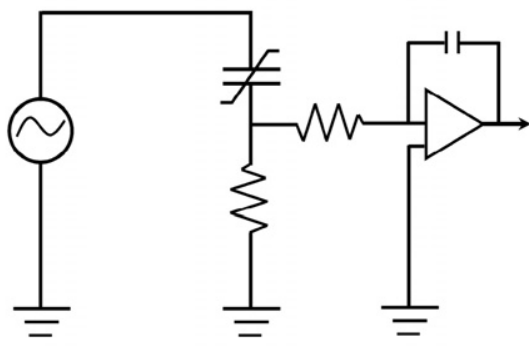

(b)

Figure 3. (a) Original Sawyer-Tower circuit and (b) modified Sawyer-Tower circuit for polarization hysteresis loop measurement.
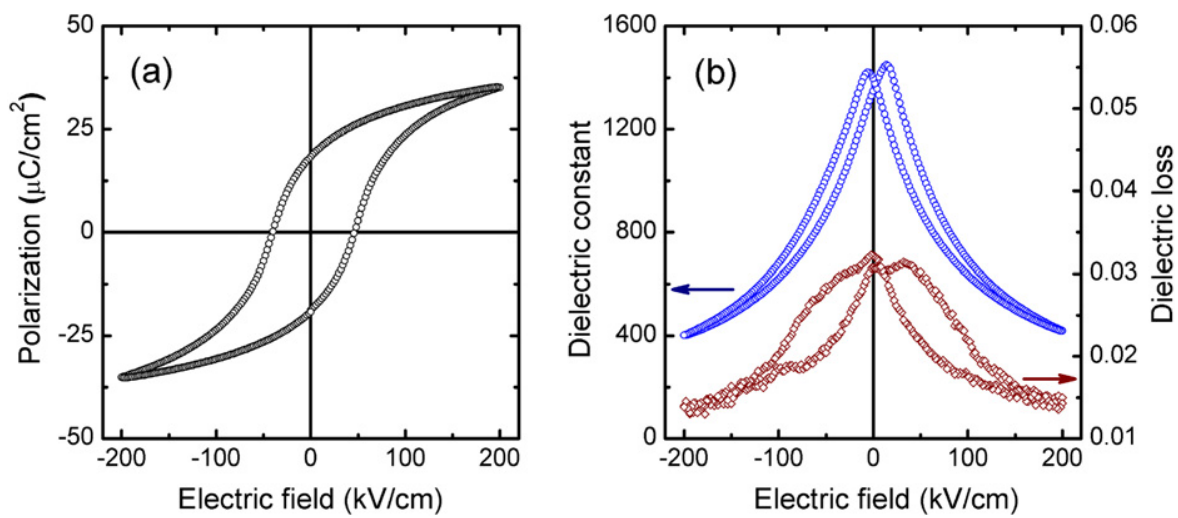

Figure 4. (a) Polarization hysteresis $(P-E)$ loop, and (b) dielectric constant $(\varepsilon-E)$ and dielectric loss (tan $\delta-E)$ curves, measured on a $500-\mu \mathrm{m}$-diameter PZT film membrane actuator. The thickness of PZT film is $1 \mu \mathrm{m}$.

amplitude and $1 \mathrm{kHz}$ frequency, using a ferroelectric tester system (aixACCT TF-2000 Analyzer).

Together with remnant polarization, the dielectric constant and dielectric loss are also used to evaluate the quality of thin film deposition. The dielectric constant and dielectric loss are calculated from the capacitance measurement [34]. In this paper, a Süss MicroTech PM300 manual probe-station equipped with a Keithley 4200 Semiconductor characterization system was used for the capacitance measurement. The capacitance-electric field $(C-E)$ measurement was performed at an ac signal of $1 \mathrm{kV} \mathrm{cm} \mathrm{cm}^{-1}$ and $10 \mathrm{kHz}$ frequency with the dc bias sweeping from -200 to $+200 \mathrm{kV} \mathrm{cm}^{-1}$ and then back to $-200 \mathrm{kV} \mathrm{cm}^{-1}$.

Figure 4 shows the remnant polarization $\left(P_{\mathrm{r}}\right)$, dielectric constant $(\varepsilon)$ and dielectric loss $(\tan \delta)$ of $1-\mu$ m-thick PZT film. The values of $P_{\mathrm{r}}, \varepsilon$ and $\tan \delta$ are $18.4 \mu \mathrm{C} \mathrm{cm}^{-2}, 1360$ and 0.03 , respectively. They are in good agreement with the reported values [35], indicating good quality PZT films are formed.

\subsection{Piezoelectric displacement and resonance responses}

The Polytech MSA-400 micro-system analyzer was used for the analysis and visualization of the structural vibration in piezoelectric membranes. A fully integrated optical microscope with a scanning laser Doppler vibrometer (LDV) allows the measurement of the piezoelectric displacement as well as the resonant responses of piezoelectric actuators. For measuring the out-of-plane displacement of the membrane, the structure was excited by applying a sinusoidal ac-voltage of $6 V_{\mathrm{p}-\mathrm{p}}$ (peak-to-peak) at $8 \mathrm{kHz}$ frequency. This frequency is far away from the resonance frequencies of the actuators. The resonance-frequency measurement was performed at an excitation voltage of $1 V_{\text {ac }}$ in the frequency range from 0 to $2 \mathrm{MHz}$. A scheme of the LDV is presented in figure 5.

\section{Results and discussion}

The optimal electrode coverage (EC) for the maximum piezoelectric actuator displacement can be predicted through an understanding of the mechanical stress in the membrane. Mechanical stress control in suspended membranes is important, as the intrinsic stress in the membrane directly determines the sensitivity of the actuator to any applied voltage. When an electrical field is applied across the top and bottom electrodes of the piezoelectric stack, the PZT film will expand or contract in the thickness direction, and oppositely contract or expand in the plane. Since the piezoelectric stack is fixed at the silicon-PZT interface this will induce a stress that will bend the membrane [37]. In extreme situations tensile stress can cause membranes to fracture, while compressive stresses result in collapsed membranes. Figure 6 shows the mechanical stress in the $\mathrm{PZT} /$ silicon membrane. When the tensile stress is induced in the central region in the lateral direction, the compressive stress is induced in the surroundings close to the membrane 


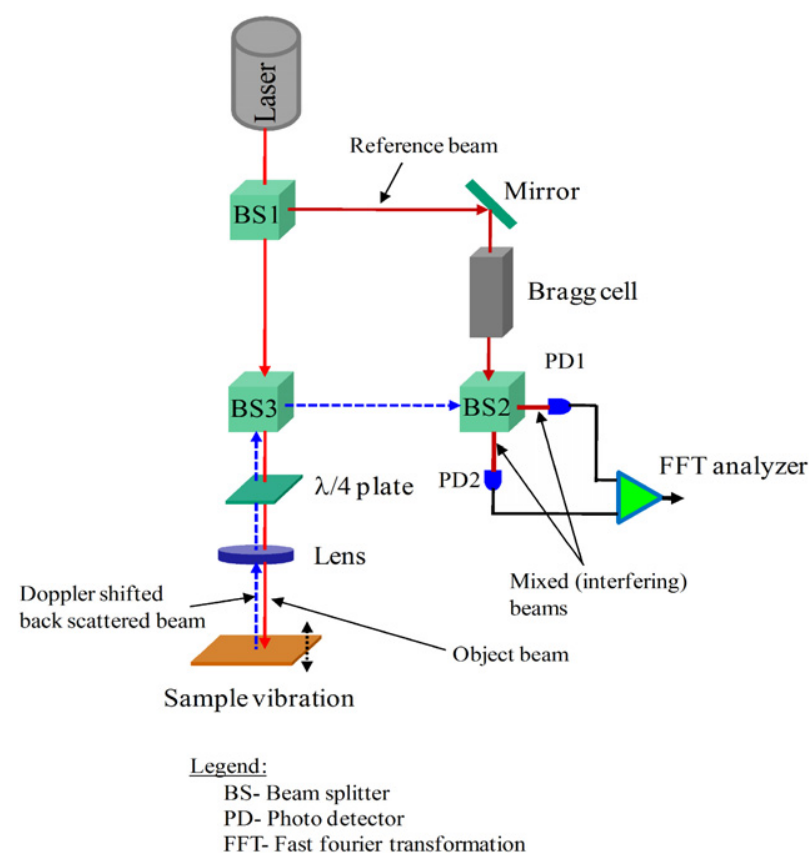

Figure 5. Scheme of the LDV [36].

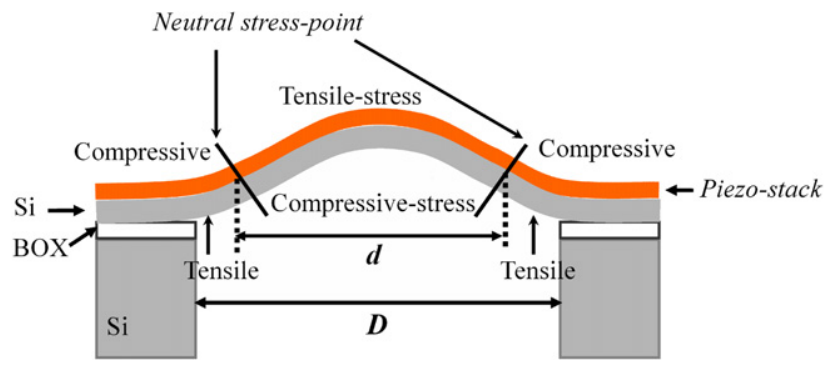

Figure 6. Schematic illustration of stress behavior at the top and the bottom surface of a PZT/Si membrane bending with fixed boundaries.

edge. The stresses in these two regions always have opposite signs to each other. A neutral-stress (or zero-stress) point exists between the two stressed regions [37]. In order to maximize the piezoelectric displacement of the membrane, the top-electrode in the piezoelectric sensors is located within the two neutral-stress points (called as the active area) to avoid opposite polarizations in the positive- and negative-stress regions canceling each other. The calculation and simulation results on the relation between piezoelectric displacement and electrode area show that there exists an optimal EC value that gives the highest displacement of the membrane [37]. The EC value can be written as:

$$
E C=\frac{\pi(d / 2)^{2}}{\pi(D / 2)^{2}}=\frac{1+v}{3+v}
$$

where $d$ and $D$ are the diameters of the top-electrode and $\mathrm{Si}$ membrane, respectively, and $v$ is the Poisson's ratio of the piezoelectric membrane.

Using the average Poisson's ratio weighted by the thickness, for the two layers, such as the PZT film $(v=0.30$, thickness: $0.5-3.0 \mu \mathrm{m})$ and the Si device layer $(v=0.28$,
Table 1. Values of material parameters used.

\begin{tabular}{lll}
\hline & Si device layer & PZT film \\
\hline$E(\mathrm{GPa})$ & $130.2[39]$ & $113.5[40]$ \\
$\rho\left(\mathrm{kg} \mathrm{m}^{-3}\right)$ & $2320[41]$ & $7700[42]$ \\
$\nu$ & $0.28[39]$ & $0.30[43,44]$ \\
$t(\mu \mathrm{m})$ & 5 & 1 \\
\hline
\end{tabular}

thickness: $5 \mu \mathrm{m}$ ), then calculated EC values of about $39 \%$ are obtained. In this calculation, the contribution of the buffer-layer and electrode layers is assumed to be negligible due to the thickness of these layers being much thinner than that of the PZT film and Si device layer. The result shows that the thickness of the PZT film and/or Si device layer does not affect the calculated EC value (see more in equation (1)), because there is not much difference between the values of Poisson's ratio for the PZT film and the Si device layer.

Figure 7(a) shows the center displacement and resonance frequency of the partially covered membrane in terms of the electrode coverage. When the EC value is reduced, the resonant spectrum will shift to a higher frequency range. The displacement corresponding to its resonance frequency first increases with increasing EC value and then decreases, reaching a maximum value at $\mathrm{EC}=36 \%$.

The next study is to find the optimized electrode coverage, where the diameters of the membrane are varied from 200 to $500 \mu \mathrm{m}$. Results of the piezoelectric displacement as a function of the EC value are shown in figures 8(a) and (b). The displacement is maximized at an EC value of $36 \%$ for membrane diameters of 300-500 $\mu \mathrm{m}$. However, the maximum displacement was achieved at a lower EC value $(25 \%)$ with the smaller membrane size $(200 \mu \mathrm{m})$. This effect can be explained by a change in the clamped boundary condition at the edge of the membrane. The clamped edge is increased and then the active-area ratio in membrane $(d / D$, see figure 6) is reduced with a decreasing membrane diameter. The decrease of active-area ratio is directly related to the increase of the measured resonance frequency, in comparison with the calculated values (see figure 8(c)), with the decreasing membrane size.

In this paper, the resonance frequency of a circular membrane is calculated as [38]:

$$
f_{\mathrm{r}}=\frac{\lambda_{n}^{2} t}{2 \pi r^{2}} \sqrt{\frac{E}{12 \rho\left(1-v^{2}\right)}}
$$

where $E$ and $v$ are the average Young's modulus and average Poisson's ratio of the membrane, respectively; $\rho$ is its average density, $t$ is its thickness and $r$ is radius of the membrane; $\lambda_{n}$ is the resonance eigenvalue, where $n$ denotes the resonance mode, i.e. first mode $\lambda_{1}=3.196$. The material parameters used for the silicon device and piezoelectric thin film are listed in table 1. In this calculation, the contribution of the buffer-layer and electrode layers is assumed to be negligible due to the thickness of these layers being much thinner than those of the PZT film and Si device layer.

Figure 9 shows the relative effect of the PZT film thickness on the membrane displacement. It is noted that the 

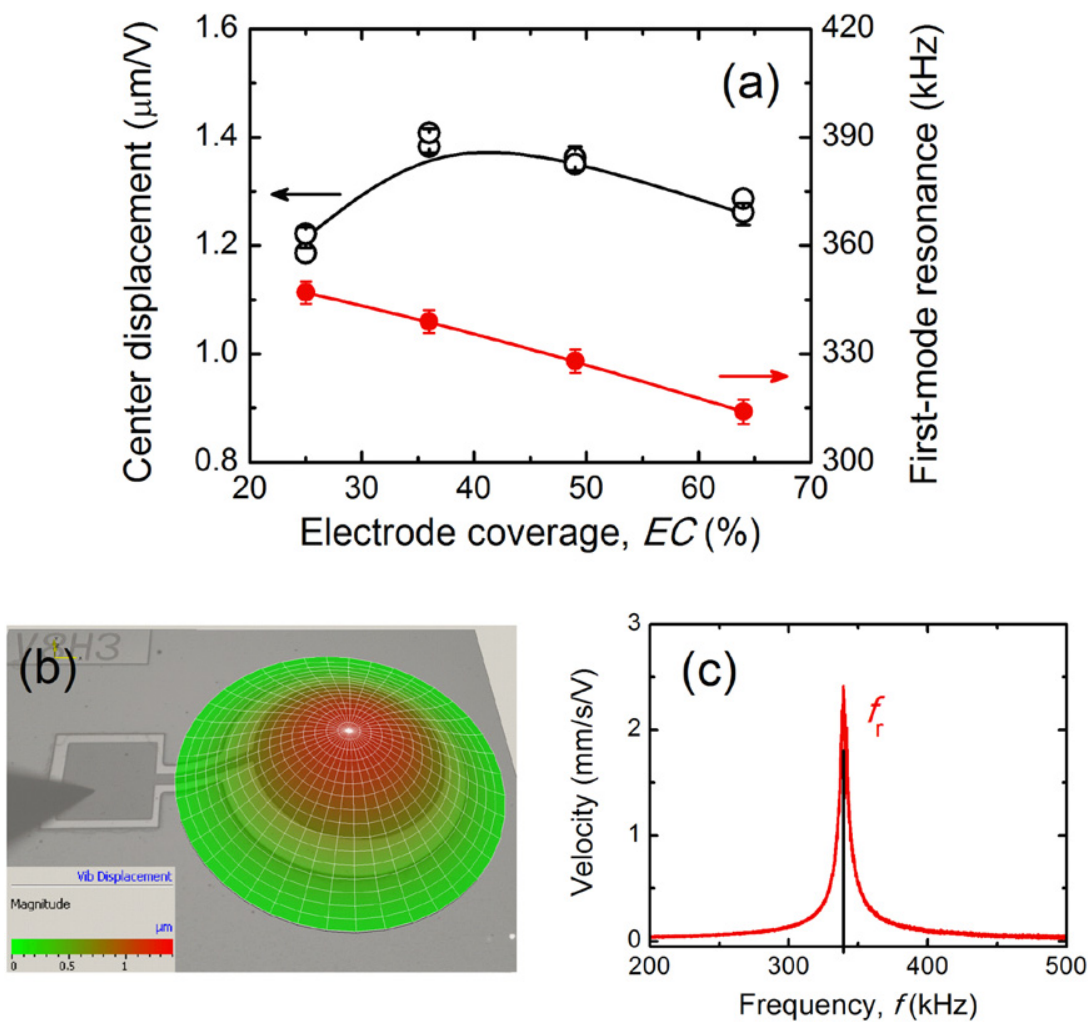

Figure 7. (a) Measured center-displacement and first-mode resonance frequency as a function of electrode coverage (EC) of $500-\mu$ m-diameter membrane actuators; (b) upward displacements at the first-mode resonance frequency $\left(f_{1}=339 \mathrm{kHz}\right)$ and (c) resonance-frequency spectrum, measured on a 500- $\mu$ m-diameter PZT film membrane actuator with EC $=36 \%$. The thicknesses of the PZT film and Si device layer are 1 and $5 \mu \mathrm{m}$, respectively.

(a)
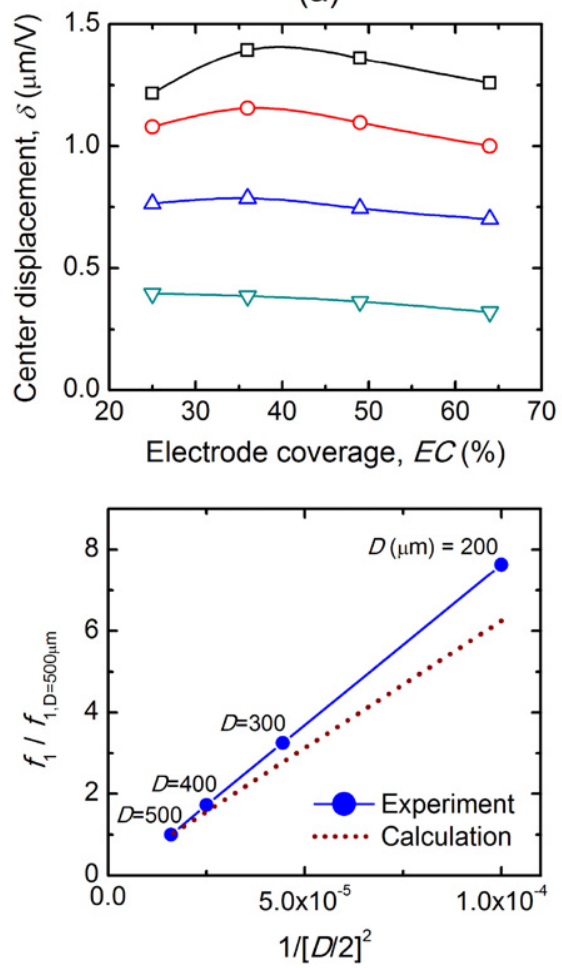

(b)

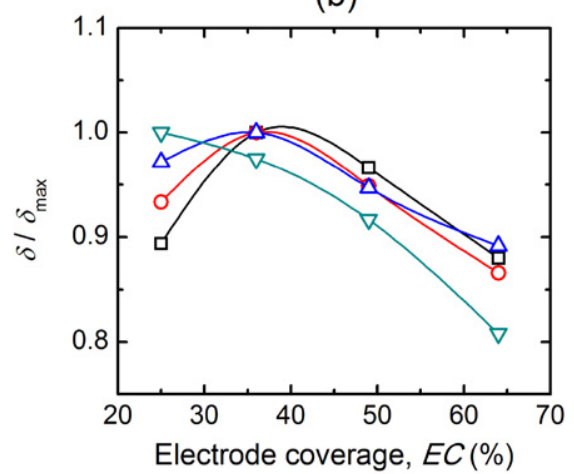

(c)

Figure 8. (a) Displacement and (b) normalized displacement $\left(\delta / \delta_{\max }\right)$, measured on different diameters of membrane actuators and as a function of electrode coverage; (c) measured (solid line) and calculated (dotted line) values of the first-mode resonance frequency versus the inverse square of the radius of the membrane actuators with $\mathrm{EC}=36 \%$. The thicknesses of PZT film and Si device layer are 1 and $5 \mu \mathrm{m}$, respectively. 

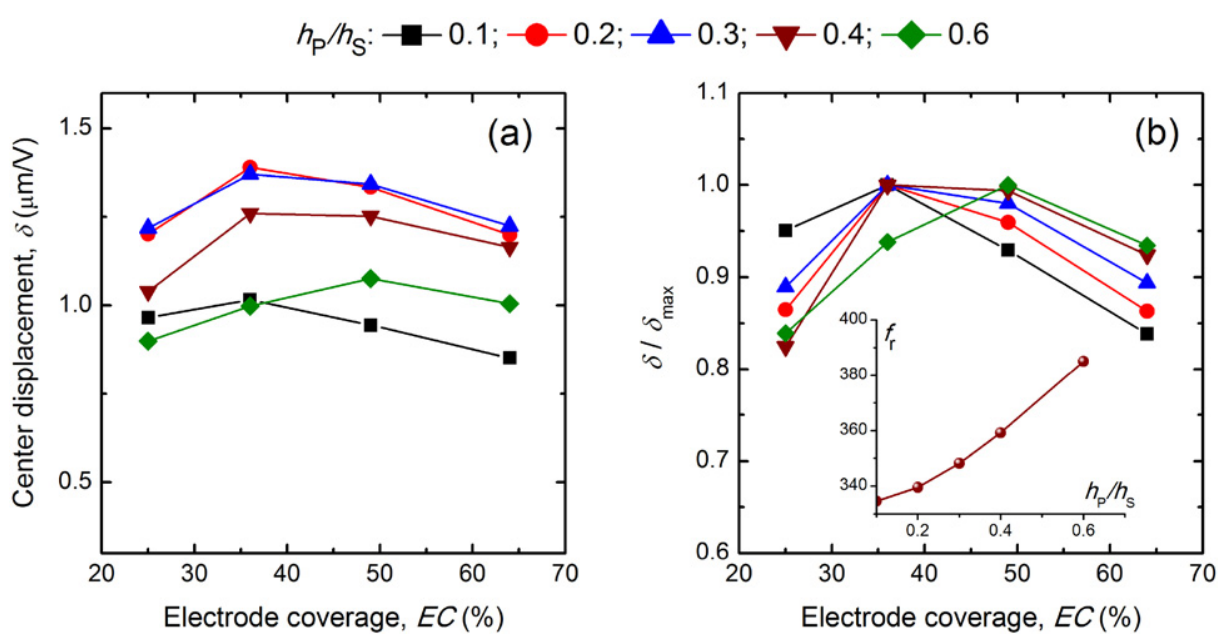

Figure 9. (a) Displacement and (b) normalized displacement $\left(\delta / \delta_{\max }\right)$, measured on membrane actuators as a function of the PZT-film/silicon-device thickness ratio $\left(h_{\mathrm{P}} / h_{\mathrm{S}}\right)$ and as a function of electrode coverage. Inset figure presents a relation between first-mode resonance frequency and the $h_{\mathrm{P}} / h_{\mathrm{S}}$ ratio, measured on membrane actuators with EC $=36 \%$. The thickness of the Si device layer is $5.0 \mu \mathrm{m}$.

membrane displacement is affected by the thickness ratio of the piezoelectric PZT film layer $\left(h_{\mathrm{P}}\right)$ and Si device layer $\left(h_{\mathrm{S}}\right)$. As shown in figure $9(\mathrm{a})$, the value of $h_{\mathrm{P}} / h_{\mathrm{S}}$ to maximize the displacement of the membrane is found to be $0.2-0.3$ (or the PZT film thickness in the range of $1.0-1.5 \mu \mathrm{m}$, while the Si device-layer thickness was held constant at $5.0 \mu \mathrm{m}$ ). This result is in good agreement with the theoretical calculation and experiment that were obtained by Muralt et al [45], in which the 0.6- $\mu \mathrm{m}$-thick PZT film was sputtered on membranes with a $2 \mathrm{~mm}$ diameter and a Si device layer of 3-6 $\mu \mathrm{m}$ in thickness. However, the optimal $h_{\mathrm{P}} / h_{\mathrm{S}}$ ratio is not constant, but is affected by the quality of the PZT film as well as the thickness of the Si device layer. With an optimal PZT film thickness, the optimized $h_{\mathrm{P}} / h_{\mathrm{S}}$ ratio for the maximized membrane displacement could be increased by decreasing the thickness of the Si device layer.

The effect of film thickness on displacement can be explained based on: (i) the contribution of the domain structure in association with the change due to the residual stress and substrate clamping; and (ii) the existence of an interfacial layer between the film and electrode [46]. The increased effective piezoelectric coefficient $d_{33 \text {,f }}$ with film thickness is caused by the reduction of the clamping effect induced by the silicon substrate [47], see figure 10 for instance, in which a $d_{33, \mathrm{f}}$ saturation value was observed at a film thickness of $1 \mu \mathrm{m}$ [46]. However, that optimized film thickness is also affected by the fabrication technique, e.g. the

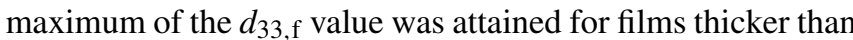
$2 \mu \mathrm{m}$ by using sol-gel method [48].

As seen in figure 9, an electrode coverage of $36 \%$ is also observed to give the maximum displacement. However, the EC value also varies with the $h_{\mathrm{P}} / h_{\mathrm{S}}$ ratio. The data shows that the optimal EC value is shifted to $49 \%$ with a $h_{\mathrm{P}} / h_{\mathrm{S}}$ ratio of 0.6. This can be explained by the decrease in the substrate clamping with increasing film thickness and leads to an increase in the active membrane area or EC value.

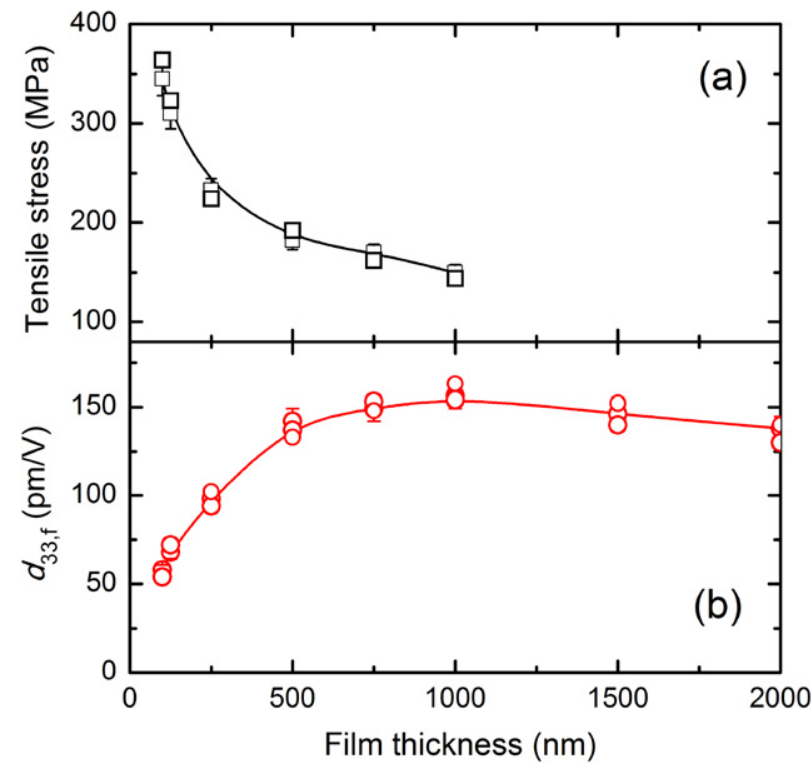

Figure 10. (a) Measured residual tensile stress and (b) effective piezoelectric coefficient of PZT film capacitors according to the film thickness [46]. The PZT films were deposited using the PLD technique.

\section{Conclusions}

Piezoelectric micro-actuators based on epitaxial all-oxide piezoelectric film stacks and $\mathrm{Si}$-membranes have been designed, fabricated, and characterized. Membrane diameter and top-electrode size were varied independently in order to understand their effects on the performance of the devices.

(i) The maximum center displacement is obtained at an optimal EC value of $36 \%$ when the membrane diameter is in the range $300-500 \mu \mathrm{m}$. With a smaller membrane size, the optimal EC is shifted towards a lower value. 
(ii) The improvement in membrane displacement is mostly due to the increase in membrane diameter rather than to the optimization of the EC.

(iii) Experiments show that the $h_{\mathrm{P}} / h_{\mathrm{S}}$ ratio to maximize the membrane displacement is found to be $0.2-0.3$ (or a thickness of PZT films in the range of $1.0-1.5 \mu \mathrm{m}$ while the thickness of $\mathrm{Si}$ device layer is held constant at $5.0 \mu \mathrm{m})$.

\section{Acknowledgments}

This work is financially supported by Smart-Mix Program (SmartPie) of the Netherlands Ministry of Economic Affairs and the Netherlands Ministry of Education, Culture and Science; and by Vietnam National Foundation for Science and Technology Development (NAFOSTED) under Grant number 103.02-2011.43. The authors also acknowledge helpful discussions with and comments from Assoc. Professor Dr L Abelmann at the Transducers Science and Technology group, University of Twente, The Netherlands.

\section{References}

[1] Fan B, Song G and Hussain F 2005 Simulation of a piezoelectrically actuated valveless micropump Smart Mater. Struct. 14 400-5

[2] Nisar A, Afzulpurkar N, Mahaisavariya B and Tuantranont A 2008 MEMS-based micropumps in drug delivery and biomedical applications Sensors Actuators B $130917-42$

[3] Cui Q, Liu C and Zha X F 2007 Study on a piezoelectric micropump for the controlled drug delivery system Microfluid. Nanofluid. 3 377-90

[4] Laser D J and Santiago J G 2004 A review of micropumps J. Micromech. Microeng. 14 R35-64

[5] Yun K S and Yoon E 2006 Micropumps for MEMS/NEMS and microfluidic systems MEMS/NEMS Handbook Techniques and Applications ed T L Cornelius (Berlin: Springer) pp 121-53

[6] Mu Y H, Hung N P and Ngoi K A 1999 Optimisation design of a piezoelectric micropump Int. J. Adv. Manuf. Technol. 15 573-6

[7] Yunas J, Johari J, Bahadorimehr A R, Gebeshuber I C and Majlis B Y 2011 Investigation of simple process technology for the fabrication of valveless micropumps $A d v$. Mater. Res. 254 211-4

[8] Brinkman E 2011 The Hidden Use of Piezo Technology in Applications All Around us (Apeldoorn: Applied Piezo Foundation) p 45

[9] Shoji S and Esashi M 1994 Microflow devices and systems J. Micromech. Microeng 4 157-71

[10] Shoji S 1998 Fluids for sensor systems Microsystem Technology in Chemistry and Life Science ed A Manz and H Becker (Berlin: Springer) pp 163-88

[11] Gass V, van der Schoot B H, Jeanneret S and de Rooij N F 1994 Integrated flow-regulated silicon micropump Sensors Actuators A 43 335-8

[12] Scott J F and Paz de Araujo C A 1989 Ferroelectric memories Science 246 1400-5

[13] Kondo M, Maruyama K and Kurihara K 2002 Epitaxial ferroelectric thin films on silicon substrates for future electronic devices Fujitsu Sci. Tech. J. 38 46-53

[14] Kim C J, Yoon D S, Lee J S, Choi C G, Lee W J and No K 1994 Electrical characteristics of (100), (111), and randomly aligned lead zirconate titanate thin films J. Appl. Phys. 76 7478-82

[15] Akai D, Yokawa M, Hirabayashi K, Matsushita K, Sawada K and Ishida M 2005 Ferroelectric properties of sol-gel delivered epitaxial $\mathrm{Pb}\left(\mathrm{Zr}_{x}, \mathrm{Ti}_{1-x}\right) \mathrm{O}_{3}$ thin films on Si using epitaxial $\gamma-\mathrm{Al}_{2} \mathrm{O}_{3}$ layers Appl. Phys. Lett. 86202906

[16] Cho J, Anderson M, Richards R, Bahr D and Richards C 2005 Optimization of electromechanical coupling for a thin-film PZT membrane: I. Modeling J. Micromech. Microeng. 15 1797-803

[17] Cho J, Anderson M, Richards R, Bahr D and Richards C 2005 Optimization of electromechanical coupling for a thin-film PZT membrane: II. Experiment J. Micromech. Microeng. 15 1804-9

[18] Choi H S, Anderson M J, Ding J L and Bandyopadhyay A 2010 A two-dimensional electromechanical composite plate model for piezoelectric micromachined ultrasonic transducers (pMUTs) J. Micromech. Microeng. 20015013

[19] Hishinuma Y and Yang E H 2006 Piezoelectric unimorph microactuator arrays for single-crystal silicon continuous-membrane deformable mirror J. Microelectromech. Syst. 15 370-9

[20] Hata T, Kawagoe S, Zhang W, Sasaki K and Yoshioka Y 1998 Proposal of new mixture target for PZT thin films by reactive sputtering Vacuum $\mathbf{5 1}$ 665-71

[21] Bouregba R, Sama N, Soyer C and Remiens D 2009 Analysis of size effects in $\mathrm{Pb}\left(\mathrm{Zr}_{0.54} \mathrm{Ti}_{0.46}\right) \mathrm{O}_{3}$ thin film capacitors with platinum and $\mathrm{LaNiO}_{3}$ conducting oxide electrodes J. Appl. Phys. 106044101

[22] Tokumitsu E, Ueno S, Nakamura R I and Ishiwara H 1995 Characterization of $\mathrm{Pb}\left(\mathrm{Zr}_{x} \mathrm{Ti}_{1-x) \mathrm{O}_{3}}\right.$ films prepared by vacuum evaporation method Integr. Ferroelectr. 7 215-23

[23] Park B E, Shouriki S, Tokumitsu E and Ishiwara H 1998 Fabrication of $\mathrm{PbZr}_{x} \mathrm{Ti}_{1-x \mathrm{O}_{3}}$ films on $\mathrm{Si}$ structures using $\mathrm{Y}_{2} \mathrm{O}_{3}$ buffer layers Japan. J. Appl. Phys. 37 5145-9

[24] Pan C Y, Chen Y L and Tsai D S 2002 Synthesis and properties of lead zirconate titanate thin films via metalorganic chemical vapor deposition J. Mater. Res. 17 1536-42

[25] Otani Y, Okamura S and Shiosaki T 2004 Recent developments on MOCVD of ferroelectric thin films J. Electroceram. 13 15-22

[26] Han H, Zhong J, Kotru S, Padmini P, Song X Y and Pandey R K 2006 Improved ferroelectric property of $\mathrm{LaNiO}_{3} / \mathrm{Pb}\left(\mathrm{Zr}_{0.2} \mathrm{Ti}_{0.8}\right) \mathrm{O}_{3} / \mathrm{LaNiO}_{3}$ capacitors prepared by chemical solution deposition on platinized silicon Appl. Phys. Lett. 88092902

[27] Vu H N, Le M V, Bui H T and Nguyen M D 2009 Improvement of electrical property for $\mathrm{Pb}\left(\mathrm{Zr}_{0.53} \mathrm{Ti}_{0.47}\right) \mathrm{O}_{3}$ ferroelectric thin film deposited by sol-gel method on SRO electrode J. Phys.: Conf. Ser. 187012063

[28] Singh S K, Lees M R, Singh R K and Palmer S B 2002 Growth of $\mathrm{SrRuO}_{3}$ thin films on $\mathrm{MgO}$ substrates by pulsed laser ablation J. Phys. D: Appl. Phys. 35 2243-6

[29] Bornand V, Trolier-McKinstry S, Takemura K and Randall C A 2000 Orientation dependence of fatigue behavior in relaxor ferroelectric- $\mathrm{PbTiO}_{3}$ thin films $J$. Appl. Phys. 87 3965-72

[30] Nguyen M D, Nazeer H, Karakaya K, Pham S V, Steenwelle R, Dekkers M, Abelmann L, Blank D H A and Rijnders G 2010 Characterization of epitaxial $\mathrm{Pb}(\mathrm{Zr}, \mathrm{Ti}) \mathrm{O}_{3}$ thin films deposited by pulsed laser deposition on silicon cantilevers J. Micromech. Microeng. 20085022

[31] Dekkers M, Nguyen M D, Steenwelle R, te Riele P M, Blank D H A and Rijnders G 2009 Ferroelectric properties of epitaxial $\mathrm{Pb}(\mathrm{Zr}, \mathrm{Ti}) \mathrm{O}_{3}$ thin films on silicon by control of crystal orientation Appl. Phys. Lett. 95012902

[32] Sawyer C B and Tower C H 1930 Rochelle salt as a dielectric Phys. Rev. 35 269-73 
[33] Tsui Y T, Hinderaker P D and McFadden F J 1968 New ferroelectric hysteresis curve tracer featuring compensation and virtual sample grounding Rev. Sci. Instrum. 39 1423-4

[34] Nguyen M D 2010 Ferroelectric and piezoelectric properties of epitaxial PZT films and devices on silicon PhD Thesis University of Twente, The Netherlands

[35] Kearney D R 2013 Optimization of pulsed laser deposited piezoelectric thin films for microelectromechanical systems BSc Thesis University Honors College, Oregon State, USA

[36] Esposito E 2008 Laser Doppler vibrometry Handbook of the Use of Lasers in Conservation and Conservation Science ed M Schreiner, M Strlič and R Salimbeni (Brussels: COST Office)

[37] Olsen J K 2007 Piezoelectric components in microfluidic devices MSc Thesis Technical University of Denmark, Denmark

[38] Hong E 2004 Surface micromachined peristaltic pumps using lead zirconate titanate film $P h D$ Thesis The Pennsylvania State University, Pennsylvania, USA

[39] Brantley W A 1973 Calculated elastic constants for stress problems associated with semiconductor devices $J$. Appl. Phys. 44 534-5

[40] Nazeer H 2012 Thin films on cantilevers PhD Thesis University of Twente, The Netherlands

[41] Madou M J 2011 Fundamentals of Microfabrication and Nanotechnology (Manufacturing Techniques for Microfabrication and Nanotechnology) 3rd edn, vol 2 (Boca Raton, FL: CRC Press, Taylor \& Francis) p 496
[42] Sherrit S, Wiederick H D, Mukherjee B K and Sayer M 1997 An accurate equivalent circuit for the unloaded piezoelectric vibrator in the thickness mode J. Phys. D: Appl. Phys. 30 2354-63

[43] Shepard J F Jr, Moses P J and Trolier-McKinstry S 1998 The wafer flexure technique for the determination of the transverse piezoelectric coefficient $\left(d_{31}\right)$ of PZT thin films Sensors Actuators A 71 133-8

[44] Tuchiya T, Itoh T, Sasaki G and Suga T 1996 Preparation and properties of piezoelectric lead zirconate titanate thin films for microsensors and microactuators by sol-gel processing J. Ceram. Soc. Japan 104 159-63

[45] Muralt P, Kholkin A, Kohli M and Maeder T 1996 Piezoelectric actuation of PZT thin-film diaphragms at static and resonant conditions Sensors Actuators A 53 398-404

[46] Nguyen M D, Dekkers M, Vu H N and Rijnders G 2013 Film-thickness and composition dependence of epitaxial thin-film PZT-based mass-sensors Sensors Actuators A 199 98-105

[47] Kim D M, Eom C B, Nagarajan V, Ouyang J, Ramesh R, Vaithyanathan V and Schlom D G 2006 Thickness dependence of structural and piezoelectric properties of epitaxial $\mathrm{Pb}\left(\mathrm{Zr}_{0.52} \mathrm{Ti}_{0.48}\right) \mathrm{O}_{3}$ films on $\mathrm{Si}$ and $\mathrm{SrTiO}_{3}$ substrates Appl. Phys. Lett. 88142904

[48] Lian L and Sottos N R 2000 Effects of thickness on the piezoelectric and dielectric properties of lead zirconate titanate thin films J. Appl. Phys. 87 3941-9 